\title{
Investigation of Cervical Intervertebral Discs before and after Surgery for Adolescent Idiopathic Scoliosis
}

Kosuke Takimura $^{1^{*}}$, Noriaki Kawakami ${ }^{1}$, Tetsuya Ohara ${ }^{1}$, Taichi Tsuji ${ }^{1}$, Yoshitaka Suzuki ${ }^{1}$, Toshiki Saito ${ }^{1}$, Ayato Nohara ${ }^{1}$, Ryoji Tauchi $^{1}$, Tsuneo Takebayashi $^{2}$ and Toshihiko Yamashita ${ }^{2}$

${ }^{1}$ Department of Orthopaedic and Spine Surgery, Meijo Hospital, Nagoya, Japan

${ }^{2}$ Sapporo Medical University School of Medicine, Sapporo, Japan

"Corresponding author: Kosuke Takimura, Department of Orthopaedic and Spine Surgery, Meijo Hospital, Nagoya, Japan, Tel: 819076425002; E-mail: k13t1108@yahoo.co.jp

Rec Date: September 08, 2017; Acc Date: September 15, 2017; Pub Date: September 19, 2017

Copyright: ( 2017 Takimura K, et al. This is an open-access article distributed under the terms of the Creative Commons Attribution License, which permits unrestricted use, distribution, and reproduction in any medium, provided the original author and source are credited.

\begin{abstract}
Objective: The aim of this study was to assess the changes in MRI signal intensities of cervical intervertebral discs following corrective surgery in patients with Adolescent Idiopathic Scoliosis (AIS) who were postoperatively followed up for longer than 10 years.
\end{abstract}

Introduction: Sagittal alignment of the cervical spine secondarily may change after corrective surgery in patients with AIS. No previous studies have evaluated MRI images to investigate postoperative changes in cervical intervertebral discs considering cervical alignment that may be influenced by surgical intervention for AIS.

Methods: Eighty-nine patients with AIS who underwent MRI scans preoperatively and 10 years postoperatively following surgery. Control group of volunteers with no neck pain underwent MRI on the cervical spine. Both groups were evaluated the signal intensity and morphology of intervertebral discs (nucleus pulposus) on sagittal T2weighted MRI images of the $\mathrm{C} 2 / 3$ to $\mathrm{C} 7 / \mathrm{T} 1$ discs. Nucleus pulposus signal intensity was assessed using that of the spinal cord as reference and classified as "normal" (N), dehydration (DH), degeneration (DG), and "intermediate" (IM).

Results: The AIS group included 89 cases and the control group included 35 cases. Comparison of pre- and postoperative signal intensity in the AIS group indicated that signal intensity significantly decreased postoperatively for all intervertebral discs $(\mathrm{P}<0.01)$ except for the $\mathrm{C} 7 / \mathrm{T} 1$ disc. On the other hand, decreased signal intensity accounted for over half of the discs in the control group, except for the C7/T1 disc; however, no statistically significant differences were observed between the postoperative and the control groups.

Conclusion: Postoperative cervical disc intensity had significantly reduced in comparison with preoperative values. However, no statistically significant differences were observed between the control and postoperative group with regard to reduced signal intensity. Pre-to postoperative sagittal cervical alignment changes may have affected the changes in signal intensity.

Keywords: Adolescent idiopathic scoliosis; Surgical treatment; Cervical intervertebral disc; Dehydration; MRI

\section{Introduction}

Water content of intervertebral discs reportedly decreases with age, resulting in subsequent reductions in the signal intensity of the nucleus pulposus on T2-weighted magnetic resonance imaging (MRI) [1-3]. In this context, the term "dehydration" (DH) refers to a state in which the signal intensity reduces on T2-weighted MRI, without any accompanying "degeneration" (DG) [1-3]. In 1995, Buckwarter et al. reported a mechanism responsible for reductions in proteoglycan levels and water content of intervertebral discs [4].

Despite the high clinical significance of these findings, there is no clear indication regarding when the reduced signal intensity of intervertebral disc changes from the DH to DG state or whether DH has already changed to DG. A correlation has been reported between reduced intervertebral disc intensity and age. However, varying degrees of reduced intervertebral disc intensity can be detected in young, healthy patients with no symptoms and who may be affected by cervical kyphosis or posture.

Some patients with adolescent idiopathic scoliosis (AIS) exhibit cervical kyphosis; this has been clinically confirmed using X-rays of the entire spine [5-7]. Furthermore, this is strongly associated with reduced thoracic kyphosis in AIS [8]. Other studies have reported that sagittal cervical alignment secondarily changes after corrective surgery for scoliosis [8-12]. However, no previous studies have used MRI to investigate postoperative changes in cervical intervertebral discs and cervical alignment in patients with AIS. Therefore, we here aimed to investigate the changes in MRI signal intensities of cervical intervertebral discs following corrective surgery for AIS by considering cervical alignment changes in patients who underwent long-term follow-up for at least 10 years postoperatively. 


\section{Materials and Methods}

Our study sample included 89 patients with AIS who underwent MRI scans preoperatively and at 10 years postoperatively following corrective surgery before 20 years of age, and between 1995 and 2004, at the Department of Orthopaedics and Spine Surgery, Meijo Hospital, Nagoya, Japan. All surgeries were performed by a single surgeon (NK). All subjects were female, and the mean age at the time of surgery was $14.2 \pm 1.9$ years (11 to 19 years). The mean age at postoperative followup MRI scans was $24.8 \pm 2.4$ years ( 21 to 30 years), and the mean postoperative follow-up observation period was 12 years $(9$ years 3 months to 17 years 6 months).

Further, a control group of volunteers with no cervical pain underwent cervical MRI scans under the same conditions as those for patients with AIS, and the presence or absence of any changes in signal intensity was compared. All subjects in the control group were female, and the mean age was $25.8 \pm 2.5$ years ( 21 to 29 years). For both groups, we evaluated the signal intensity and morphology of intervertebral discs (nucleus pulposus) on sagittal T2-weighted MRI of the $\mathrm{C} 2 / 3$ to $\mathrm{C} 7 / \mathrm{T} 1$ discs.

The signal intensity of the nucleus pulposus was assessed using that of the spinal cord as reference. Results indicating higher signal intensity in the nucleus pulposus than in the spinal cord were defined as "normal" $(\mathrm{N})$, those indicating comparable signal intensity were defined as $\mathrm{DH}$, and those indicating lower signal intensity than the spinal cord and reduced cervical intervertebral disc height were defined as DG. Patients in whom spinal cord signal intensity was between DH and DG with absence of intervertebral disc narrowing were classified as "intermediate" (IM).

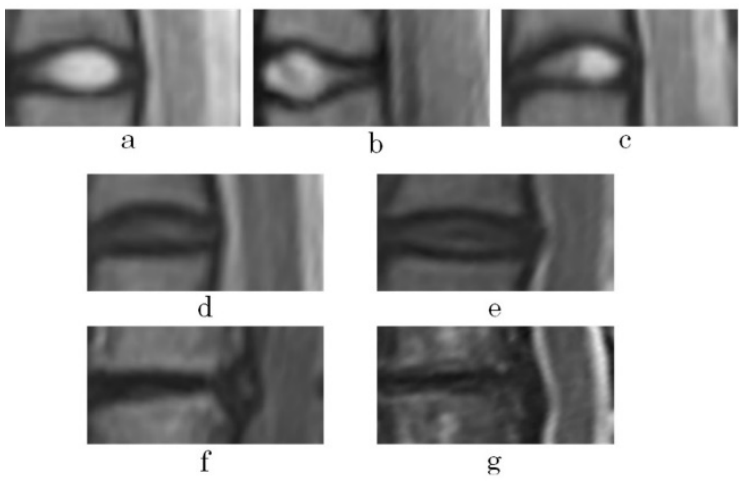

Figure 1: Classification of disc degeneration with intensity (MRI T2 weighed images) in cervical spine. A) Nc (Normal central): Higher signal intensity than spinal cord, nucleus pulposus in center, B) $\mathrm{Na}$ (Normal anterior): Higher signal intensity than spinal cord, nucleus pulposus anterior, C) $\mathrm{Np}$ (Normal posterior): Higher signal intensity than spinal cord, nucleus pulposus posterior, D) DH (Dehydration): Same signal intensity as spinal cord, E) IM (Intermediate): Between $\mathrm{DH}$ and $\mathrm{DG}$, not accompanied by intervertebral disc narrowing, F) DG (Degeneration) B(-): Lower signal intensity than spinal cord, decreased disc height, no bony changes, G) DG (Degeneration) B (+): Lower signal intensity than spinal cord, decreased disc height, bony changes present.

Furthermore, $\mathrm{N}$ was classified into three types: "N central" $(\mathrm{Nc})$, where the nucleus pulposus was located in the center of the intervertebral disc, "N anterior" (Na), where the nucleus pulposus had deviated in the anterior direction, and "N posterior" ( $\mathrm{Np}$ ), where the nucleus pulposus had deviated in the posterior direction. DG was classified into DG B (-) or (+), indicating the absence or presence of bony changes, such as bone spicules, respectively (Figures 1A-1G).

In the scoliosis group, the scoliosis Cobb angle and sagittal curvature, both in C2-7 and T2-12, were measured on whole spine Xrays obtained pre- and postoperatively; cervical alignment was assessed on the basis of classifications reported by Miaou et al. [13]. $\mathrm{Nc}, \mathrm{Na}$, and $\mathrm{Np}$ patients were designated as the healthy group $(\mathrm{N}$ group), whereas DH (D1) and IM (D2) patients were designated as the reduced signal intensity group (D group). Pre- and postoperative images were compared in the scoliosis group, and postoperative images of the scoliosis groups were compared with those of the control group.

The MRI equipment used included the MRH-500 0.5 T (Hitachi, Tokyo, Japan) prior to 1997, Visart 1.5 T (Toshiba Medical, Tochigi, Japan) from 1997 onward, EXCELART Vantage XGV 1.5 T (Toshiba Medical, Tochigi, Japan) from 2007 onward, and EXCELART Vantage Atlas 1.5 T (Toshiba Medical, Tochigi, Japan) from 2008 onward.

\section{Error testing for the classification of intervertebral changes in T2-weighted MRI scans}

Before commencing this study, we tested determination errors in the classification of changes in intervertebral disc intensity, where five spinal experts classified cervical intervertebral disc intensity on T2weighted MRI scans of cervical vertebrae from 10 randomly selected patients twice with 1-week intervals. Inter- and intra-examiner errors were measured on the basis of their interpretations of the readings. The results indicated that there were no statistically significant inter- $(0.1 \%$ Kruskal-Wallis, SPSS) or intra-observer $(0.1 \%$ Mann-Whitney U, SPSS) (Table 1) errors with regard to C2/3-C7/1 level.

\begin{tabular}{|c|c|c|c|}
\hline \multicolumn{2}{|c|}{ Interobserver error } & \multicolumn{2}{c|}{ Intraobserver error } \\
\hline Level & P & Level & P \\
\hline C2/3 & 0.091 & C2/3 & 0.156 \\
\hline C3/4 & 0.919 & C3/4 & 0.428 \\
\hline C4/5 & 0.301 & C4/5 & 0.189 \\
\hline C5/6 & 0.304 & C5/6 & 0.028 \\
\hline C6/7 & 0.544 & C6/7 & 0.028 \\
\hline C7/T1 & 0.026 & C7/T1 & 1 \\
\hline \multicolumn{2}{|c|}{$(0.1 \%$ Kruskal-Wallis $)$} & \multicolumn{2}{c|}{$(0.1 \%$ Mann-Whitney U) } \\
\hline
\end{tabular}

Table 1: Inter- and intraobserver error in evaluation of disc intensity.

\section{Results}

\section{Surgery details and overall image of patients}

The surgical procedures performed in the 89 patients with AIS included anterior spine fusion (37 patients, 41\%), posterior spine fusion (39 patients, $44 \%$ ), and anterior release+posterior spine fusion (13 patients, 15\%). Lumbar modifier and thoracic sagittal modifier, in relation to the Lenke classification, of all 89 patients are shown in Table 2 . 
Citation: Takimura K, Kawakami N, Ohara T, Tsuji T, Suzuki Y, et al. (2017) Investigation of Cervical Intervertebral Discs before and after Surgery for Adolescent Idiopathic Scoliosis. J Spine 6: 389. doi:10.4172/2165-7939.1000389

Page 3 of 6

\begin{tabular}{|c|c|c|c|c|c|}
\hline \multicolumn{2}{|c|}{ Lenke type } & \multicolumn{2}{c|}{ Lumbar modifer } & \multicolumn{2}{c|}{ Sagittal modifier } \\
\hline 1 & 49 & A & 40 & N & 54 \\
\hline 2 & 19 & B & 11 & + & 6 \\
\hline 3 & 3 & C & 38 & - & 29 \\
\hline 4 & 0 & - & - & - & - \\
\hline 5 & 49 & - & - & - & - \\
\hline 6 & 14 & - & - & - & - \\
\hline 6 & 4 & - & - & - & - \\
\hline
\end{tabular}

Table 2: Lenke classification in 89 patients.

Lenke type 1 was the most common curve type, lumbar modifier A and $\mathrm{C}$ accounted for approximately the same proportion of patients, and two-thirds of patients exhibited the normal range of thoracic kyphosis. The Upper Instrumented Vertebrae (UIV) differed between patients depending on the type and degree of curvature. This ranged from T2 to T7 for thoracic scoliosis, with T4 being the most common, whereas the UIV for thoracolumbar vertebral/lumbar vertebral scoliosis included T11 or T12 of lower thoracic vertebrae in most patients (Figure 2).

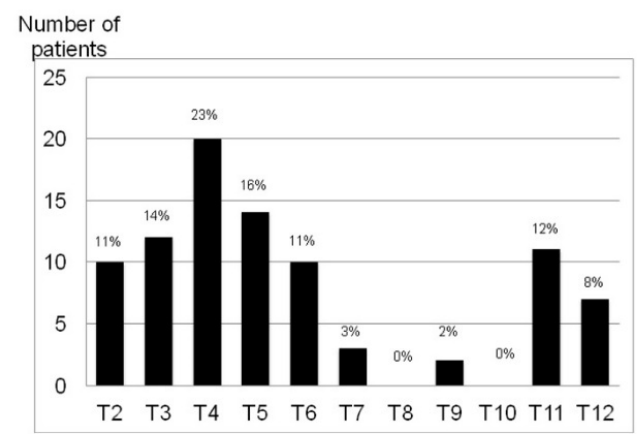

Figure 2: Location of upper instrumented vertebrae.

The mean degree of scoliosis was $58.6^{\circ} \pm 28.3^{\circ}$ (45-86) preoperatively, $17.4^{\circ} \pm 9.2^{\circ}(2-36)$ immediately postoperatively, and $22.9^{\circ} \pm 11.0^{\circ}(11-35)$ at 10 years postoperatively. The correction rate and loss were $71.1 \% \pm 10.8 \%(48-96)$ and $5.2 \pm 4.8^{\circ}(0-22)$, respectively (Figure 3).

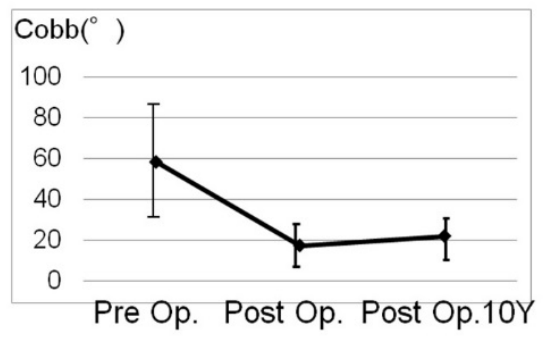

Figure 3: Changes in pre and postoperative scoliosis Cobb angles.

Sagittal curvature measurements for the C2-7 angle showed a mean preoperative value of $10.7^{\circ} \pm 10.5^{\circ}(-14-38)$ and a mean 10 -year postoperative value of $8.3^{\circ} \pm 10.4^{\circ}(-23-50)$. For the T2-12 angle, the mean preoperative value was $23.0^{\circ} \pm 11.4^{\circ}(2-50)$, and the mean 10 year postoperative value was $28.5 \pm 9.0^{\circ}(2-52)$. There were large individual variations observed among patients, with no apparent fixed trends.

\section{Evaluation of vertebral disc intensity}

The AIS group included 89 patients (534 intervertebral discs), while the control group included 35 (210 intervertebral discs). Because all subjects in this study were female and their ages approximately ranged from 10 to 30 years, no patients did not exhibit intervertebral discs that could be classified as "DG."

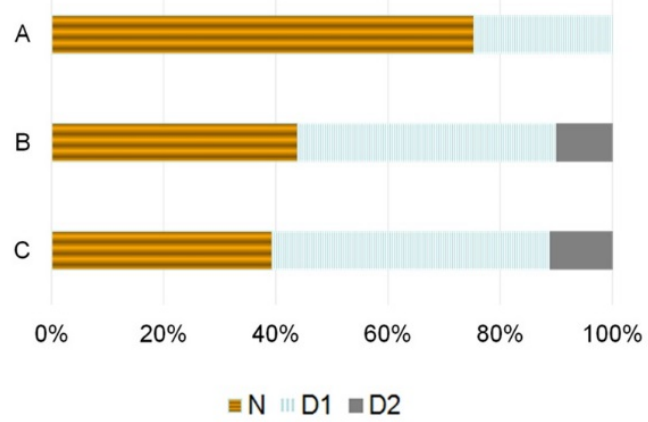

Figure 4: Percentages of disc degenerations based on MRI findings (A) preoperative and (B) postoperative AIS group, and (C) Control group (N: Normal; D1: Dehydration; D2: Intermediate).

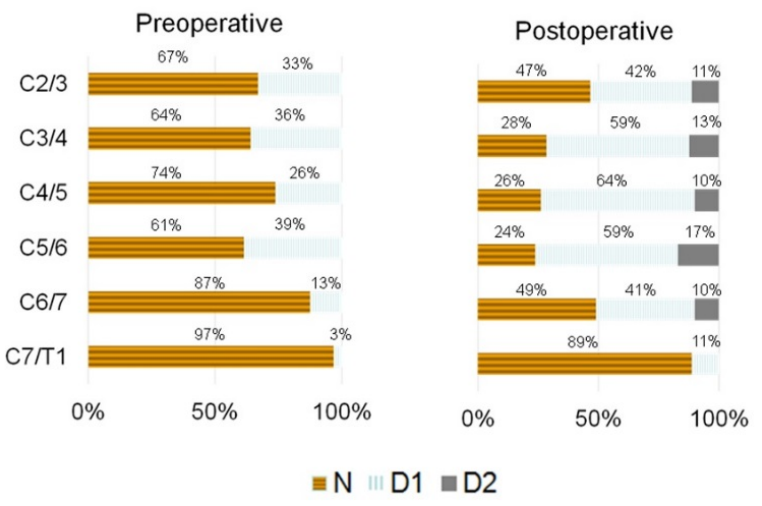

Figure 5: Types of disc intensity based on MRI findings for each disc level in the AIS group. Upper graphs: preoperative types of disc intensity in each disc segment Lower graphs: postoperative types of disc intensity in each disc segment (N: Normal; D1: Dehydration; D2: Intermediate).

Therefore, to make differences among patients more apparent, the $\mathrm{N}$ group was classified into three stages $(\mathrm{Nc}, \mathrm{Na}$, and $\mathrm{Np}$ ), depending on the degree of intervertebral disc intensity, whereas the $\mathrm{D}$ group was divided into D1 (DH) and D2 (IM). We then compared the pre- and 
postoperative values, followed by subsequent comparison of these with the values of the control group.

With regard to changes in intervertebral disc intensity by disc level, the proportion of patients that were D1 postoperatively increased for all intervertebral discs. D2, which was not observed for any patient preoperatively, increased by $\geq 10 \%$ for all intervertebral discs, except for the $\mathrm{C} 7 / \mathrm{T} 1$ disc (Figures 5).

Our results showed that the intervertebral disc intensity in the AIS group was $75 \%$ for preoperative $\mathrm{N}$ and $25 \%$ for $\mathrm{D} 1(\mathrm{DH})$; no other signal intensity changes were observed. Postoperatively, N was 44\%, D1 (DH) was $46 \%$, and D2 (IM) was $10 \%$. This indicated that D2, which could not be preoperatively observed, could now be examined postoperatively. The corresponding percentages for the control group were $40 \%, 49 \%$, and $11 \%$ for $\mathrm{N}, \mathrm{D} 1(\mathrm{DH})$, and $\mathrm{D} 2(\mathrm{IM})$, respectively (Figures 4A-4C).

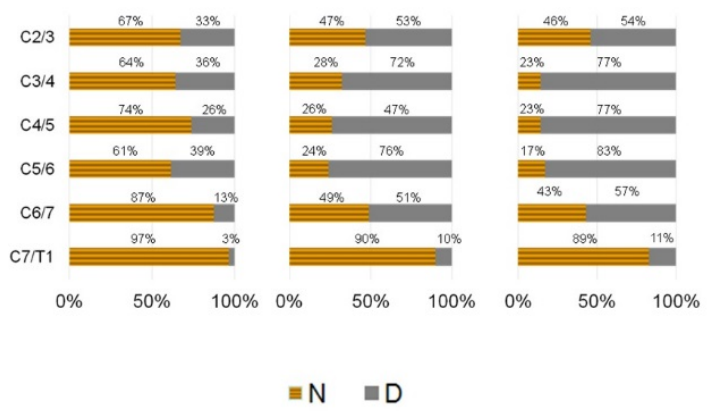

Figure 6: Comparison of types in disc intensity for each disc segment A) AIS group: pre-operative, B) AIS group: postoperative, C) Control group (N: Normal; D: Dehydration).

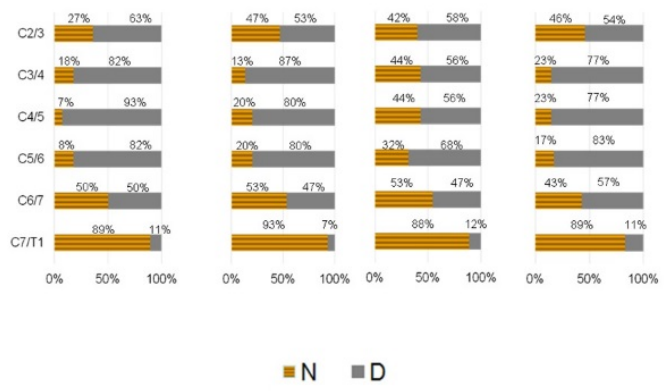

Figure 7: Comparison of types in disc intensity for each disc segment in terms of postoperative cervical alignment. A) Patients with alignment getting worse postoperatively, B) Patients with their alignment getting better postoperatively, C) Patients with almost the same alignment, D) control group (N: Normal, D: Dehydration).

Comparison of pre- and postoperative values for the AIS group indicated that D (i.e., reduced signal intensity) had postoperatively increased for all intervertebral discs, with a statistically significant difference noted for all discs, except for the C7/T1 disc (Figures 6A-6C).
On the other hand, D accounted for over half of the intervertebral discs in the control group, except for the C7/T1 disc. However, no statistically significant differences were observed between the postoperative and control groups (Figures 7A-7D).

\section{Cervical alignment evaluation}

Cervical alignment was evaluated in 89 patients using Miaou classifications. Data indicated that the kyphosis type accounted for approximately half of the patients both pre- (52 patients) and postoperatively (44 patients). This was sequentially followed by the straight and sigmoid types; these trends remained the same pre- and postoperatively. Cervical alignment changed in 40 patients (45\%) postoperatively (Table 3 ), with 28 changing to the sigmoid or kyphosis type and exhibiting an increased kyphosis angle; 15 patients changed to the lordosis direction, and 34 exhibited no changes in alignment postoperatively.

\begin{tabular}{|c|c|c|c|c|c|}
\hline Post Op. & \multirow{2}{*}{ Lordosis } & Straight & Sigmoid & Kyphosis & Total \\
\cline { 1 - 5 } Pre-Op. & & 6 & 1 & 1 & 9 \\
\hline Lordosis & 1 & 9 & 4 & 4 & 21 \\
\hline Straight & 4 & 0 & 3 & 3 & 7 \\
\hline Sigmoid & 1 & 8 & 6 & 36 & 52 \\
\hline Kyphosis & 2 & 23 & 14 & 44 & 89 \\
\hline Total & 8 & & & & \\
\hline
\end{tabular}

Table 3: Changes of cervical sagittal alignment in 89 patients.

Comparison of the signal intensity changes between the case and control groups showed no significant correlation between alignment changes and intervertebral disc intensity changes $(\mathrm{P}>0.05$, MannWhitney U, SPSS) (Figure 7).

\section{Discussion}

Nohara et al. reported that lumbar intervertebral disc DG was noted in $61.5 \%$ AIS patients 10 years after corrective surgery and that such DG was common in the L5/S disc. In addition, few studies have investigated intervertebral disc degenerative changes in the lumbar vertebrae after corrective surgery for thoracic scoliosis using MRI findings and reported similar results, in that intervertebral disc degenerative changes increased to a greater extend in L5/S than in the control group.

The only studies investigating intervertebral disc degenerative changes of the cervical spine before and after scoliosis surgery in adults were conducted using X-rays, not using MRI images. Elisha et al. [14] compared 48 adult idiopathic scoliosis patients who underwent long fusion from Th10 to the sacrum, with a control group of 38 patients. They reported that degenerative changes of the cervical spine significantly increased in the surgical group, according to X-ray findings, indicating that fusion to the thoracic spine may accelerate degenerative changes of the cervical spine.

Although few studies have investigated cervical intervertebral disc changes with MRI, they have all focused on degenerative changes that occur once patients reach adulthood, resulting in classification of intervertebral disc intensity changes as a marker for age-related DG [15-19]. The natural course of cervical intervertebral DG has been 
investigated using MRI findings. Okada et al. [15] reported that reduced MRI signal intensity was noted after 10 years in $59.6 \%$ cervical intervertebral discs and that a significant correlation was noted with age alone. No significant correlations were observed with gender, smoking, alcohol, sports, or body mass index.

The present study focused on MRI signal intensity changes in the intervertebral discs of adolescent females; no previous studies have compared pre- and postoperative MRI findings of adolescent to young adult patients. Therefore, we created original classifications for intervertebral disc intensity changes at the beginning of this study and evaluated inter- and intra-observer errors. When creating these classifications, the fact that overall signal intensity can be freely altered with manual contrast-brightness control on MRI images proved to be an issue. To resolve this, we evaluated intervertebral disc intensity on the basis of whether it was lower or higher than spinal cord intensity under any imaging conditions.

The characteristics of MRI scans of AIS patients who underwent surgery included 1) a marked trend for reduced signal intensity in almost all cervical intervertebral discs (C2/3-C6/7), except C7/T1, at 10 years postoperatively and small postoperative signal intensity changes in the lowest cervical intervertebral disc (C7/T1) (Figures 5 and 6) no clear differences in reduced signal intensity for any intervertebral discs between the postoperative and control groups (Figure 2). When the frequencies of disc DG after corrective fusion were compared between the lumbar and cervical discs, lumbar intervertebral disc DG onset after corrective surgery for AIS was clearly higher for the L5/S disc $[13,20]$, while there were no such differences for the cervical intervertebral disc in our study.

These differences may be explained by the following. First, more postoperative changes in sagittal alignment were noted for the cervical spine than for the lumbar spine, suggesting that intervertebral disc intensity changes are a mechanical issue caused by corrective fusion surgery and could be easily influenced by sagittal alignment. In fact, Hwang et al. [8] reported a correlation between postoperative thoracic kyphosis in AIS patients and cervical alignment and that thoracic hypokyphosis was a risk factor for cervical kyphosis. In addition, several reports have indicated that the three-dimensional correction of thoracic scoliosis in AIS patients had a significant impact on cervical alignment $[8-12,21,22]$. Second, because intervertebral discs of the cervical spine are smaller and narrower than those of the lumbar spine, it may have been difficult to discern signal intensity changes on MRI in a precise manner. Third, as stated above, it is impossible to distinguish between intervertebral disc DG and reversible intervertebral disc $\mathrm{DH}$; therefore, the results may include both conditions. In the present study, sagittal alignment improved in 15 of 89 patients and was exacerbated in 28 of 89 patients. The absence of a statistically significant difference could be accounted for by the fact that these pre- to postoperative sagittal alignment changes and the reversible changes in reduced intervertebral disc intensity may have affected the results. Therefore, it may be too early to conclude that corrective surgery in AIS patients does not affect cervical intervertebral disc degenerative changes 10 years postoperatively and that all changes observed were within the range of age-related changes alone.

The present study had the following limitations: 1) subjects included all Lenke types, and the operative range differed by patients; 2) the sample size was too small to investigate operative range by Lenke type; 3) MRI equipment differed over the observation course, and there were differences in resolution; 4) clinical symptoms such as nape pain were not sufficiently investigated; and 5) the follow-up period of 10 years was too short, and the mean age of subjects was 24.8 years; therefore, the subjects were very young for investigating intervertebral disc DG. Thus, future much longer-term studies examining the clinical significance of signal intensity changes in cervical intervertebral discs are required.

\section{Conclusion}

We used MRI to evaluate changes in cervical intervertebral discs of AIS patients undergoing long-term postoperative follow-up. The results of this study indicated that postoperative cervical intervertebral disc intensity had significantly reduced in comparison with preoperative values. However, no statistically significant differences were observed between the control and postoperative group with regard to reduced signal intensity. Moreover, pre- to postoperative sagittal alignment changes in cervical vertebrae may have affected the changes in signal intensity. Therefore, future long-term studies examining the clinical significance of signal intensity changes in cervical intervertebral discs are required.

\section{References}

1. Raesekhi A, Babaahmadi A, Assadsangabi R, Nabavizadeh SA (2006) Clinical manifestations and MRI findings of patients with dehydrated lumber disc herniation. Acad Radiol 13: 1485-1489.

2. Luoma K, Vehmas T, Riihimaki H, Raininko R (2001) Disc height and signal intensity of the nucleus pulposus on magnetic resonance imaging as indicators of lumber disc degeneration. Spine 26: 680-686.

3. Sether LA, Yu S, Haughton VM, Fischer ME (1990) Intervertebral disk: Normal age-related changes in MR signal intensity. Radiology 177: 385-388.

4. Buckwalter JA (1995) Aging and degeneration of the human intervertebral disc. Spine 20: 1307-1314.

5. Cochran T, Irstam L, Nachemson A (1983) Long-term anatomic and functional changes in patients with adolescent idiopathic scoliosis treated by Harrington rod fusion. Spine 8: 576-583.

6. Rechtman AM, Borden AGB, Gershon-Cohen J (1961) The lordotic curve of the cervical spine. Clin Orthop 20: 208-216.

7. Winter RB, Lovell WW, Moe JH (1975) Excessive thoracic lordosis and loss of pulmonary function in patients with idiopathic scoliosis. J Bone Joint Surg Am 57: 972-977.

8. Hwang S, Samdani A, Tantorski M, Cahill P, Nydick J, et al. (2011) Cervical sagittal plane decompensation after surgery for adolescent idiopathic scoliosis. J Neurosurg Spine 15: 491-496.

9. Moskowitz A, Moe JH, Winter RB, Binner H (1980) Long-term follow-up of scoliosis fusion. J Bone Joint Surg Am 62: 364-376.

10. Canavese F, Turcot K, De Rossa V, De Coulon G, Kaelin A (2011) Cervical sagittal alignment variations following posterior spinal fusion and instrumentation for adolescent idiopathic scoliosis. Eur Spine J 20: 1141-1148.

11. Hilibrand AS, Tannenbaum DA, Granziano GP, Loder RT, Hensinger RN (1995) The sagittal alignment of cervical spine in adolescent idiopathic scoliosis. J Pediatr Orthop 15: 627-632.

12. Yann PC, Gergi S, Valérie MP, Sauleau EA, Steib JP (2015) Cervical sagittal alignment in idiopathic scoliosis treated by posterior instrumentation and in situ bending. Spine 40: 419-427.

13. Nohara A, Kawakami N, Tsuji T, Ohara T, Saito T, et al. (2013) Intervertebral disc degeneration during postoperative follow-up more than 10 years after correction surgery in idiopathic scoliosis: Comparison between patients with and without surgery. J Spine Res 4: 1651-1655.

14. Ofiram E, Garvey TA, Schwender JD, Wroblewski JM, Winter RB (2009) Cervical degenerative changes in idiopathic scoliosis patients who underwent long fusion to the sacrum as adults: incidence, severity, and evolution. J Orthopaed Traumatol 10: 27-30. 
Citation: Takimura K, Kawakami N, Ohara T, Tsuji T, Suzuki Y, et al. (2017) Investigation of Cervical Intervertebral Discs before and after Surgery for Adolescent Idiopathic Scoliosis. J Spine 6: 389. doi:10.4172/2165-7939.1000389

Page 6 of 6

15. Okada E, Matsumoto M, Ichihara D, Chiba K, Toyama Y, et al. (2009) Aging of the cervical spine in healthy volunteers. Spine 34: 706-712.

16. Viikari-Juntura E, Raininko R, Videnman T (1989) Evaluation of cervical disc degeneration with ultralow field MRI and discography. An experimental study on cadavers. Spine 14: 616-619.

17. Lehto IJ, Tertti MO, Komu ME, Paajanen HE, Tuominen J, et al. (1994) Age-related MRI changes at $0.1 \mathrm{~T}$ in cervical discs in asymptomatic subjects. Neuroradiol 36: 49-53.

18. Matsumoto M, Fujimura Y, Suzuki N, Nishi Y, Nakamura M, et al. (1998) MRI of the cervical intervertebral discs in asymptomatic subjects. J Bone and Joint Surg Br 80: 19-24.

19. Lee TH, Kim SJ, Lim SM (2013) Prevalence of disc degeneration in asymptomatic Korean subjects. Part2: Cervical spines. J Korean Neurosurg 53: 89-95.
20. Green DW, Lawhorne TW, Widmann RF, Kepler CK, Ahern C, et al. (2011) Long-term magnetic resonance imaging follow-up demonstrates minimal transitional level lumbar disc degeneration after posterior spinal fusion for adolescent idiopathic scoliosis. Spine 36: 1948-1954.

21. Edgar MA, Mehta MH (1988) Long-term follow-up of fused and unfused idiopathic scoliosis. J Bone Joint Surg Br 71: 712-716.

22. Ilharreborde B, Vidal C, Skalli W, Mazda K (2013) Sagittal alignment of the cervical spine in adolescent idiopathic scoliosis treated by posteromedial translation. Eur Spine J 22: 330-337. 